\title{
A Fully Portable TCP Implementation Using XFSMs
}

\author{
Giuseppe Bianchi \\ CNIT/University of Rome "Tor \\ Vergata" \\ Giacomo Belocchi \\ CNIT/University of Rome "Tor \\ Vergata"
}

\author{
Michael Welzl \\ University of Oslo \\ Marco Faltelli \\ CNIT/University of Rome "Tor \\ Vergata"
}

\author{
Angelo Tulumello \\ CNIT/University of Rome "Tor \\ Vergata" \\ Salvatore Pontarelli \\ CNIT/University of Rome "Tor \\ Vergata"
}

\begin{abstract}
XTRA (XFSM for TRAnsport) is a first step towards "codeonce-port-everywhere" transport protocols. XTRA's platformagnostic programming abstraction, based on an extended finite state machine formalization of a desired transport layer task, is amenable not only to SW engines, but can be directly executed in CPU-less custom HW, thus permits to harness FPGA-based NICs' offloading opportunities without any re-coding effort. We experimentally demonstrate that XTRA enables us to port a customized TCP implementation across three completely different environments (HW proofof-concept on a NetFPGA board, User-space SW over Linux' Open Data Plane, and NS3 emulator).
\end{abstract}

\section{CCS CONCEPTS}

- Networks $\rightarrow$ Transport protocols; $\bullet$ Hardware $\rightarrow$ Networking hardware;

\section{KEYWORDS}

Offloading, Data Plane Programmability, Hardware Acceleration, TCP

\section{ACM Reference Format:}

Giuseppe Bianchi, Michael Welzl, Angelo Tulumello, Giacomo Belocchi, Marco Faltelli, and Salvatore Pontarelli. 2018. A Fully Portable TCP Implementation Using XFSMs. In Proceedings of Posters-Demos '18: Posters and Demos (Posters-Demos '18). ACM, New York, NY, USA, 3 pages. https://doi.org/10.1145/3234200.3234237

Permission to make digital or hard copies of all or part of this work for personal or classroom use is granted without fee provided that copies are not made or distributed for profit or commercial advantage and that copies bear this notice and the full citation on the first page. Copyrights for components of this work owned by others than ACM must be honored. Abstracting with credit is permitted. To copy otherwise, or republish, to post on servers or to redistribute to lists, requires prior specific permission and/or a fee. Request permissions from permissions@acm.org.

Posters-Demos '18, August 20-25, 2018, Budapest, Hungary

(C) 2018 Association for Computing Machinery.

ACM ISBN 978-1-4503-5915-3/18/08_..\$15.00

https://doi.org/10.1145/3234200.3234237

\section{INTRODUCTION}

The question addressed in this demo is the following. Is there any useful "code-once-port-everywhere" platform-independent programming abstraction sufficiently complete to write a transport layer function, or even a full-fledged transport protocol, and at the same time capable to make such an abstract description of the transport protocol portable across both SW and HW platforms (e.g. FPGA-based NICs) $[4,5]$ ?

So far, portability of network functions (also) across HW platforms has been mainly addressed via HW-amenable abstractions such as OpenFlow [2] or P4 [3], and has been restricted to stateless, packet-based, processing tasks. Even stateful programming abstractions such as OpenState [1] are far from being able to support complex transport layer tasks involving buffering or scheduling-not to mention a complete TCP implementation.

Even portability "only" across SW platforms has been somewhat disregarded to date. Indeed, the transport layer is rich with protocols that are hand-customized for various use cases (TCP, UDP, MPTCP, SCTP, QUIC, to name but a few). The only thing that seems to be interchangeable is the congestion control algorithm (Linux and FreeBSD have "pluggable congestion control" frameworks), such that congestion controls are also separately customized (PCC, Remy, BBR, Cubic, DCTCP, DCQCN, etc). These implementations are silos, to be re-done when porting code, and efficient hardware usage requires deep customization per system. HotCocoa [7] and the "Congestion Control Plane" (CCP) [8] make congestion control deployment more flexible and efficient, but both of these proposals are strictly focused on congestion control only, while our focus is on a complete protocol.

\section{INTRODUCING XTRA}

What would it take to make the transport layer truly programmable-such that transport protocols could be implemented once, in one appropriate representation, and run everywhere? We found that suitably extended Finite State Machines (XFSMs), already proven successful in stateful flow processing tasks if equipped with technical means and primitives to describe and manage time, buffer space, packet generation, and arithmetic operations, are a good abstraction 
model to also formalize transport protocols. The advantage of XFSMs stems from their simplicity and amenability to both hardware and software implementation. In a software implementation, the use of XFSMs adds only a little overhead with respect to the normal operations of standard programming languages. Moreover, being XFSMs Turing complete [9], as long as the actions implemented support an adequate set of primitives, the abstraction does not limit the computational possibilities. We developed an XFSM execution engine "XTRA" (XFSM for TRAnsport) which offers an abstraction to define transport protocols which makes them both platform independent and efficient to execute. Having ported XTRA to three extremely different platforms (OpenDataPlane SW, FPGA HW, NS3 emulator), we demonstrate its benefits via a fully portable XFSM-based TCP implementation. The demonstration will show that our TCP implementation can run on all of these platforms without changing a single line of TCP code. The main elements of XTRA are summarized below:

Small set of elementary "bricks". This first requirement is somewhat straightforward and largely accepted. Many successful network programming technologies build upon the idea of composing a comprehensive network operation via basic and mutually independent building blocks.

Explicit stateful operation modeling. One of the key elements of XTRA is that it explicitly provides an upfront way to formally describe a non-trivial state evolution such as that of a TCP connection, thus involving many packets, buffer information, and comprising several state parameters continuously updated (RTT, congestion window, etc.).

Time management. Transport protocols require the management of complex events involving time, such as triggering a stored packet transmission at a later time, as well as to support primitives as retransmissions or pacing; XTRA provides a flexible interface for time management programming.

\section{TIMER-BASED TCP}

TCP's connection (Listen, SYN-Sent, SYN-Received, etc.) and congestion control (Slow Start, Congestion Avoidance, Fast Retransmit / Fast Recovery) state machines as well as the Reno congestion window update rules can be readily transformed into an XFSM. The Fast Recovery phase itself, however, is exceedingly complex. To address this, in this demo, we focused on a strictly timer-based version of TCP, which we labeled as Timer-Based TCP (TB-TCP). This choice follows the intuition of RACK [6] that uses the notion of time instead of counting segments or byte sequences to detect losses and decide to retransmit segments. When an ACK arrives that acknowledges some but not all transmitted segments, any segments that were sent earlier (by a minimum time called "reordering window") than the acknowledged segments are assumed to be lost. Not only does this allow RACK to detect more losses than other algorithms, it can effectively replace

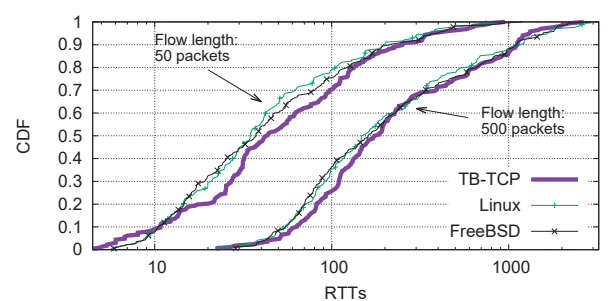

Figure 1: Completion time of the slowest flow

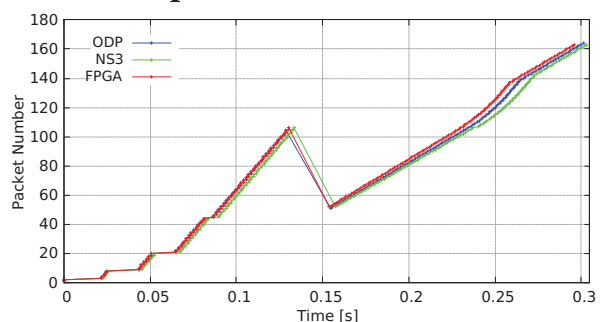

Figure 2: Time-sequence diagram of a TB-TCP flow beginning in Slow Start, then losing a packet, and concluding with our timer-based Fast Recovery phase.

other algorithms make Fast Recovery much more complex. While RACK relies on (SACK) information from DupACKs, it seems obvious that the idea of considering the amount of time that has expired since a segment was sent could also be implemented more directly, by using a physical timer (which is efficient to implement for an XFSM) for every transmitted segment. Indeed, the authors of RACK state that it "conceptually arms a (virtual) timer on every packet sent" [6]-we instead arm a physical timer on every packet sent. We tested the efficiency of the loss recovery phase using the ns-3 implementation and considering the flow completion time of the slowest of six parallel flows in approx. 400 experiments covering a large range of network conditions (varying the bottleneck capacity, RTT and the length of the bottleneck queue); Figure 1 shows that the performance of TB-TCP is very close to standard TCP, and in some cases it even outperforms both the Linux and FreeBSD TCP implementation.

\section{DEMONSTRATING PORTABILITY}

The time-sequence diagram in Figure 2 visualizes just how close the behavior of the three implementations is. This test was carried out using a single sender and receiver, interconnected with a physical link and using linux Traffic Control (TC) netem module. We see minor deviations between the three implementations, which are caused by small timing differences in how packets are clocked out. For instance, ns-3 is slightly slower at transmitting packets using emulation. The hardware implementation appears to be a little faster, which is due to the lower precision for divisions.

\section{ACKNOWLEDGMENTS}

This work has been partly funded by the EU commission in the context of the 5G-PICTURE project, GA \#762057. 


\section{REFERENCES}

[1] G. Bianchi, M. Bonola, A. Capone, C. Cascone, "OpenState: programming platform-independent stateful openflow applications inside the switch”, ACM SIGCOMM Comp. Commun. Rev. 44(2), 2014

[2] N. McKeown et. al. "OpenFlow: enabling innovation in campus networks", ACM SIGCOMM Comp. Commun. Rev. 38(2), 2008

[3] P. Bosshart et. al. "P4: Programming protocol-independent packet processors”, ACM SIGCOMM Comp. Commun. Rev. 44(3), 2014

[4] P. Shinde, A. Kaufmann, T. Roscoe, S. Kaestle, "We Need to Talk About NICs", 14th USENIX HotOS 2013

[5] Y. Le et. al. "UNO: Uniflying Host and Smart NIC Offload for Flexible Packet Processing, 2017 Symp. on Cloud Comput. (SoCC), 2017

[6] Y. Cheng, N. Cardwell, N. Dukkipati, "RACK: a time-based fast loss recovery”, 98th IETF Meeting, March 2017

[7] M.T.Arashloo, M.Ghobadi, J.Rexford, D.Walker, "HotCocoa: Hardware Congestion Control Abstractions", Proc. 16th ACM Workshop on Hot Topics in Networks (HotNets), 2017.

[8] Akshay Narayan, Frank Cangialosi, Prateesh Goyal, Srinivas Narayana, Mohammad Alizadeh, Hari Balakrishnan, "The Case for Moving Congestion Control Out of the Datapath", Proc. 16th ACM Workshop on Hot Topics in Networks (HotNets), 2017.

[9] C.-J. Wang and M. T. Liu. "A Test Suite Generation Method for ExtendedFinite State Machines Using Axiomatic Semantics Approach”, In Proc. of the XII IFIP TC6/WG6.1 Int.1 Symp. on Protocol Specification, Testing and Verification, pages 29-43, 1992. 